\title{
Avaliação da técnica de uso de dispositivos inalatórios no controle ambulatorial de asma e DPOC
}

\author{
Assessment of inhaler techniques on the control of asthma and COPD in outpatients
}

\author{
Junia Rios Garib ${ }^{1}$, Beatriz Carvalho Martins Brandão Leite ${ }^{2}$, Victor Chaves Reis ${ }^{3}$, Marcelo \\ Leonardo Araujo Campos ${ }^{4}$, Sarah Veiga Medrado ${ }^{5}$, Flávio Mendonça Andrade da Silva ${ }^{6}$
}

Garib JR, Leite BCMB, Reis VC, Campos MLA, Medrado SV, Silva FMA. Avaliação da técnica de uso de dispositivos inalatórios no controle ambulatorial de asma e DPOC / Assessment of inhaler techniques on the control of asthma and COPD in outpatients. Rev Med (São Paulo). 2018 mar.-abr.;97(2):120-7.

RESUMO: INTRODUÇÃO: Asma e Doença Pulmonar Obstrutiva Crônica (DPOC) são doenças prevalentes no Brasil e seu controle é realizado com dispositivos inalatórios (DI), cuja técnica de uso apresenta sérias dificuldades. Esse fator atinge negativamente o controle da doença e aumenta os custos para o sistema de saúde. Assim, é fundamental uma melhoria na sustentabilidade do tratamento. Atualmente, não se dispõe de dados de análise da técnica de DI em Belo Horizonte, o que justifica a realização desse trabalho. OBJETIVO: Avaliar a prevalência de erros na técnica de uso de DI em pacientes portadores de asma ou DPOC, acompanhados no Ambulatório de Pneumologia da Faculdade Ciências Médicas - MG. MÉTODOS: Estudo transversal experimental no qual foram avaliados 95 pacientes em uso de um ou mais dispositivos inalatórios. RESULTADOS: A prevalência de erros foi de 94,9\%, sendo maior nos DIs pressurizados $(98,7 \%)$ em comparação aos dispositivos em pó (88,8\%). CONCLUSÃO: A prevalência de erros na técnica de uso de DI no Ambulatório de Pneumologia da Faculdade Ciências Médicas - MG é semelhante à observada em outros estudos, sendo importante sempre orientar o paciente sobre o uso na primeira prescrição, assim como reavaliá-lo periodicamente, uma vez que esses erros podem ser corrigidos.

Descritores: Inaladores de pó seco; Inaladores dosimetrados; Asma; Doença pulmonar obstrutiva crônica.

ABSTRACT: INTRODUCTION: Asthma and Chronic Obstructive Pulmonary Disease (COPD) are prevalent diseases in Brazil. The control of the patients is performed with inhalational devices, whose usage presents serious obstacles. This factor negatively affects the control of the disease and increases the costs for the health system. An improvement in the sustainability of the treatment is fundamental. Currently, there is no data available for the inhalational devices technique in Belo Horizonte, which motivates this work. OBJECTIVE: To evaluate the prevalence of errors in the technique of inhalational devices use in patients followed due to asthma or COPD in the Ambulatório Ciências Médicas (ACM). METHODS:

Trabalho foi realizado no Ambulatório Ciências Médicas, Ciências Médicas de Minas Gerais, Belo Horizonte, MG, Brasil.

Pesquisa financiada pela FAPEMIG.

1. Professora da Disciplina de Pneumologia da Faculdade Ciências Médicas de Minas Gerais, Belo Horizonte, MG, Brasil. ORCID: orcid.org/00000002-4339-1182. Email: juniagarib@hotmail.com.

2. Acadêmica de Medicina da Faculdade Ciências Médicas de Minas Gerais, Belo Horizonte, MG, Brasil. ORCID: orcid.org/0000-0002-1690-0308. Email: beatrizcmbleite@gmail.com.

3. Acadêmico de Medicina da Faculdade Ciências Médicas de Minas Gerais, Belo Horizonte, MG, Brasil. ORCID: orcid.org/0000-0003-3811-6175. Email: victor.creis@yahoo.com.br.

4. Acadêmico de Medicina da Faculdade Ciências Médicas de Minas Gerais, Belo Horizonte, MG, Brasil. ORCID: orcid.org/0000-0001-5002-4877. Email: marcelo leocampos@hotmail.com.

5. Acadêmica de Medicina da Faculdade Ciências Médicas de Minas Gerais, Belo Horizonte, MG, Brasil. ORCID: orcid.org/0000-0002-7055-1639. Email: sarah_nese@hotmail.com.

6. Professor da Disciplina de Pneumologia da Faculdade Ciências Médicas de Minas Gerais, Belo Horizonte, MG, Brasil. ORCID: orcid.org/0000-00026263-3159. Email:. mendoncafla@gmail.com.

Endereço para correspondência: Junia Rios Garib: Rua Santa Catarina, 1036 Apt. 703. Lourdes. Belo Horizonte, MG, Brasil. CEP: $30170-080$. Email: juniagarib@hotmail.com. 
Garib JR, et al. Avaliação da técnica de uso de dispositivos inalatórios no controle ambulatorial de asma.

Experimental cross-sectional study that evaluated 95 patients undergoing medical supervision at ACM with diagnosis of asthma or COPD, in use of one or more inhalation devices. RESULTS: The prevalence of errors observed in the ACM was $98.7 \%$ in the Pressurized DI (IPre) and $88.8 \%$ in the DP type (IPo); with overall prevalence of errors of $94.9 \%$. CONCLUSION: The prevalence of errors in the usage of inhalational devices in ACM is similar to that suggested in other studies, and it is important to periodically reassess, since these errors can be corrected easily. In addition, the importance of demonstration by the prescriber at the time of the first prescription is emphasized.

Keywords: Dry powder inhalers; Metered dose inhalers; Asthma; Pulmonary disease, chronic obstructive.

\section{INTRODUÇÃO}

A sma e Doença Pulmonar Obstrutiva Crônica (DPOC) são doenças bastante prevalentes atualmente, afetando até 300 milhões de pessoas no mundo inteiro ${ }^{1}$. Um estudo multicêntrico na América Latina sobre a prevalência de DPOC indicou uma variação entre 2,5\% e $7,1 \%$ na população acima de $40 \operatorname{anos}^{2}$. Os medicamentos inalatórios são os principais métodos de controle de ambas as doenças, sobretudo na fase de manutenção ${ }^{3}$.

Os inaladores de pó (IPo) são considerados de manejo mais simples, quando comparados aos Inaladores Pressurizados (IPre), pois a ativação se dá junto à inspiração ${ }^{4}$. Além disso, aqueles demonstram resultados significativos em estudos clínicos, em relação à distribuição do medicamento no pulmão e a melhora dos pacientes ${ }^{5}$, embora em até $90 \%$ dos casos ocorra erro na técnica do uso desses dispositivos ${ }^{6}$.

A técnica de uso de cada um dos dispositivos pode ser avaliada segundo check-lists específicos e validados ${ }^{6}$.

Além de a técnica ser difícil ao primeiro contato, a aplicação correta decai com mais de 2 anos de uso do dispositivo ${ }^{7}$, evidenciando a necessidade de revisão periódica $^{8}$, através de explanação verbal e demonstração física ${ }^{3}$.

Portanto, devido à alta prevalência de erros técnicos detectados durante o uso de dispositivos inalatórios, esse estudo foi realizado a fim de verificar se os nossos pacientes também apresentam algum tipo de erro e se a prevalência é concordante com a de outros estudos.

\section{MATERIAL E MÉTODOS}

O estudo foi realizado no ambulatório de pneumologia da Faculdade de Ciências Médicas de Minas Gerais, no período de março de 2016 a maio de 2017. Participaram do estudo 95 pacientes portadores de asma ou DPOC em uso de medicação oferecida por dispositivos inalatórios, após assinatura do Termo de Consentimento Livre Esclarecido (TCLE). O diagnóstico, os níveis de controle e gravidade e o tratamento foram baseados nos critérios estabelecidos pelas Diretrizes Internacionais GOLD $^{9}$ e GINA ${ }^{10} 2016$.

O projeto foi aprovado pelo Comitê de Ética em Pesquisa Ciências Médicas - MG (CEPCM-MG), em 10/03/2016 (CAAE: 52749315.8.0000.5134). Todos os dados foram registrados em formulários e fichas padronizados para análise estatística subsequente.

Foram incluídos pacientes com idade superior a 18 anos, de ambos os sexos e que concordaram em participar da pesquisa. Aqueles com idade inferior a 18 anos, ou com distúrbios mentais e cognitivos, e em uso de dispositivo com espaçador, foram excluídos, bem como aqueles que utilizavam o dispositivo pela primeira vez na data da entrevista.

Foram avaliados os seguintes dados: idade, sexo, grau de escolaridade, doença de base, tempo de tratamento, número de dispositivos já utilizados, nível de controle da doença, tipo e frequência de uso de algum dispositivo e se houve demonstração pelo médico no momento da prescrição.

Após o preenchimento dos dados, o paciente demonstrava sua técnica diária de uso com dispositivo próprio ou de amostra, que foi avaliada de acordo com os formulários de análise (Figuras 1 e 2). No caso de pacientes que faziam uso de dois dispositivos diferentes, eram realizados testes separados. A pontuação desses critérios era somada, sendo as pontuações máxima e mínima para IPre com variação entre 9 e -18 pontos; enquanto para IPo entre 6 e -6 pontos.

Em seguida, o avaliador demonstrava com dispositivo idêntico ao do indivíduo qual a técnica correta, sem assinalar o erro cometido pelo paciente. No caso de não haver qualquer erro na técnica utilizada, este passo não era realizado, sendo considerada pontuação máxima na avaliação pré e pós intervenção. Após a demonstração, era solicitado o uso do dispositivo conforme o que o paciente aprendeu e esse segundo uso também era analisado com o mesmo formulário supracitado. Nos casos em que persistia algum erro de técnica, o paciente era orientado novamente, com intuito de proporcionar melhor terapêutica, porém não reavaliado para a pesquisa. No uso de amostras, foi realizada a limpeza adequada dos dispositivos ${ }^{8}$.

As variáveis categóricas foram apresentadas como contagens e percentuais, e as numéricas como mediana \pm distância interquartílica (diferença entre o terceiro e o primeiro quartis), em virtude da natureza não normal delas, avaliadas via teste de Shapiro-Wilk. Para a comparação de grupos, utilizou-se o teste não paramétrico de Wilcoxon para amostras independentes ou pareadas, quando adequado, e a associação entre variáveis categóricas foi avaliada via teste qui-quadrado de $\mathrm{McNemar}$ ou teste exato de Fisher. A análise foi desenvolvida no programa gratuito $\mathrm{R}$ versão 3.3 .2 e foi adotado nível de significância de 5\%. 


\begin{tabular}{|c|c|c|c|}
\hline \multicolumn{4}{|c|}{ ESCORE DA TÉCNICA DO INALADOR DE PÓ (IPo) } \\
\hline \multirow{4}{*}{\multicolumn{4}{|c|}{$\begin{array}{l}\text { Nome do paciente } \\
\text { Coloque a mão no tórax do paciente } \\
\text { Solicite que ele faça uma inspiração e depois uma expiração profunda } \\
\text { Use esta observação na avaliação do critério profundidade }\end{array}$}} \\
\hline & & & \\
\hline & & & \\
\hline & & & \\
\hline \multicolumn{3}{|c|}{ Critérios } & Pontos \\
\hline \multirow[t]{2}{*}{ Preparo da dose (acionamento do inalador) } & \multicolumn{2}{|c|}{ Enado } & 0 \\
\hline & \multicolumn{2}{|c|}{ Certo } & 1 \\
\hline \multirow{4}{*}{$\begin{array}{l}\text { Expiração (antes: inspirar profundamente e depois: } \\
\text { expirar profundamente) }\end{array}$} & \multicolumn{2}{|l|}{$\begin{array}{l}\text { Não } \\
\text { Simm }\end{array}$} & 0 \\
\hline & Sim & & 1 \\
\hline & \multirow{2}{*}{ Erros } & Respiração nasal & -4 \\
\hline & & Expirar dentro do inalador & -2 \\
\hline \multirow[t]{4}{*}{ Velocidade } & \multicolumn{2}{|c|}{ Rápida $\mathrm{OU}<3 \mathrm{~s}$} & 1 \\
\hline & \multicolumn{2}{|c|}{ Lenta ou $\geq 3 \mathrm{~s}$} & 0 \\
\hline & \multirow[t]{2}{*}{ Enros } & Inspirar irregularmente & -2 \\
\hline & & Inspirar levemente & -1 \\
\hline \multirow[t]{2}{*}{ Profundidade } & \multicolumn{2}{|c|}{ Não completamente } & 0 \\
\hline & \multicolumn{2}{|c|}{ Adequadamente } & 1 \\
\hline \multirow[t]{2}{*}{ Manutenção da CPT } & \multicolumn{2}{|c|}{$<10 \mathrm{~s}$} & 0 \\
\hline & \multicolumn{2}{|c|}{10 s ou mais } & 1 \\
\hline \multirow[t]{3}{*}{ Posiçẫo da cabeça } & \multicolumn{2}{|c|}{ Ângulo $>90^{\circ}$} & 0 \\
\hline & \multicolumn{2}{|c|}{ Angulo $<90^{\circ}$} & 1 \\
\hline & & & Total \\
\hline
\end{tabular}

CPT: capacidade pulmonar total.

Fonte: Zambelli-Simões et al.

Figura1. Escore utilizado para avaliação de técnica de uso de DI do tipo IPo

\begin{tabular}{|c|c|c|c|}
\hline \multicolumn{4}{|c|}{ ESCORE DA TÉCCNICA DO INALADOR PRESSURIZADO (IP) } \\
\hline \multicolumn{4}{|c|}{$\begin{array}{l}\text { Nome do paciente } \\
\text { Coloque a mão no tórax do paciente } \\
\text { Solicite que ele faça uma inspiração e depois uma expiração profunda } \\
\text { Use esta observação na avaliação do critério profundidade }\end{array}$} \\
\hline \multicolumn{3}{|c|}{ Critérios } & Pontos \\
\hline \multirow[t]{4}{*}{ Agitaçăo (2 ou mais vezes) } & Năo & & 0 \\
\hline & Sim & & 1 \\
\hline & Erros & Não agitar & -4 \\
\hline & & Retirar tambor/canister do aplicador & -1 \\
\hline \multirow[t]{3}{*}{ Posiçūes } & Errada & & 0 \\
\hline & Certa & & 1 \\
\hline & Erros & Retirar o espaçador & -4 \\
\hline \multirow{3}{*}{$\begin{array}{l}\text { Expiração (antes: inspirar } \\
\text { profundamente e depois expirar } \\
\text { profundamente) }\end{array}$} & Sim & & 1 \\
\hline & Não & & 0 \\
\hline & Erro & Expirar dentro do espaçador & -2 \\
\hline \multirow[t]{6}{*}{ Modo de Uso } & Dentro & m o espaçador & 0 \\
\hline & Fora di & o espaçador & 1 \\
\hline & Dentro & m espaçador & 1 \\
\hline & Fora d $t$ & espaçador & 0 \\
\hline & Enos & Usar espaçador irregularmente & -2 \\
\hline & & Boca aberta, no modo dentro & -2 \\
\hline \multirow[t]{8}{*}{ Movimento (inspiração rảpida) } & Antes $\mathrm{C}$ & & 0 \\
\hline & Junto & ois do acionamento & 1 \\
\hline & Erros & Respiraçăo nasal & -8 \\
\hline & & Completar a inspiração antes do acionamento & -4 \\
\hline & & Acionamento direto na boca e năo inspirar & -4 \\
\hline & & Inspirar muito depois do acionamento & -4 \\
\hline & & Inspirar irregularmente/entrecortada & -2 \\
\hline & & Respirar superficialmente no espaçador & -1 \\
\hline \multirow[t]{2}{*}{ Velocidade } & Rápida & & 0 \\
\hline & Lenta & & 1 \\
\hline \multirow[t]{2}{*}{ Profundidade } & Insufic & & 0 \\
\hline & Suficie & & 1 \\
\hline \multirow[t]{2}{*}{ Manutençảo da CPT } & $<10 \mathrm{~s}$ & & 0 \\
\hline & 10502 & & 1 \\
\hline \multirow[t]{3}{*}{ Intervalo (entre os acionamentos) } & $2 x$ & $<60 s$ & 0 \\
\hline & & $60 \mathrm{~s}$ ou mais & 1 \\
\hline & Dar do & nais & -4 \\
\hline & & Total & \\
\hline
\end{tabular}

Fonte: Zambelli-Simões, et al.

Figura 2. Escore utilizado para avaliação de técnica de uso de DI do tipo IPre 
Garib JR, et al. Avaliação da técnica de uso de dispositivos inalatórios no controle ambulatorial de asma.

\section{RESULTADOS}

Foram avaliados no estudo 95 pacientes, sendo $72,6 \%$ portadores de asma e $27,4 \%$ de DPOC. Pacientes do sexo masculino corresponderam a $26,3 \%$, e $35,8 \%$ dos indivíduos tinham oito anos de escolaridade ou mais. A idade mediana foi $61 \pm 18$ anos, o tempo mediano de tratamento foi $7 \pm 9$ anos e os pacientes faziam uso de 2 \pm 2 dispositivos. Inaladores em pó (IPo) foram utilizados por $51,6 \%$ dos indivíduos, e os inaladores pressurizados (IPre) foram utilizados por $82,2 \%$ (Tabela 1 ).

Tabela 1 - Características dos pacientes avaliados na amostra

\begin{tabular}{|c|c|}
\hline Variáveis & $\mathrm{n}=95$ \\
\hline Sexo Masculino & $25(26,3 \%)$ \\
\hline Sexo Feminino & $70(73,7 \%)$ \\
\hline Idade (anos) & $61 \pm 18$ \\
\hline 60 anos ou mais & $53(55,8 \%)$ \\
\hline Escolaridade & $5 \pm 5$ \\
\hline 8 anos ou mais & $34(35,8 \%)$ \\
\hline \multicolumn{2}{|l|}{ Doença de base } \\
\hline Asma & $69(72,6 \%)$ \\
\hline Controlada & $23(33,3 \%)$ \\
\hline Parcialmente controlada & $24(34,8 \%)$ \\
\hline Mal controlada & $22(31,9 \%)$ \\
\hline DPOC & $26(27,4 \%)$ \\
\hline GOLD A ou B & $17(65,4 \%)$ \\
\hline GOLD C ou D & $9(34,6 \%)$ \\
\hline Tempo de tratamento (anos) & $7 \pm 9$ \\
\hline 10 anos ou mais & $38(40 \%)$ \\
\hline Asma & $8 \pm 10$ \\
\hline DPOC & $3 \pm 8$ \\
\hline $\mathrm{N}^{0}$ dispositivos utilizados & $2 \pm 2$ \\
\hline Asma & $2 \pm 2$ \\
\hline$D P O C$ & $2 \pm 1$ \\
\hline \multicolumn{2}{|l|}{ Tipo de dispositivo utilizado* } \\
\hline Inalador de Pó & $49(51,6 \%)$ \\
\hline $\mathrm{N}^{\mathrm{o}}$ vezes ao dia & $2 \pm 1$ \\
\hline Em caso de crise & $9(9,5 \%)$ \\
\hline Inalador pressurizado & $78(82,1 \%)$ \\
\hline $\mathrm{N}^{\mathrm{o}}$ vezes ao dia & $2 \pm 2$ \\
\hline Em caso de crise & $16(16,8 \%)$ \\
\hline
\end{tabular}

Houve aumento significativo nos escores de avaliação na técnica de utilização de IPo após a intervenção $(p<0,001)$. Esse aumento foi significativo em relação a todas as variáveis analisadas, com exceção dos pacientes portadores de DPOC GOLD C ou D, nos quais não houve diferença estatística $(\mathrm{p}=1,000)$ (Tabela 2).

Aumento significativo também ocorreu nos escores de avaliação na técnica de utilização de IPre após a intervenção $(\mathrm{p}<0,001)$, sendo observado também em todas as variáveis analisadas (Tabela 3 ).
Tabela 2 - Escores de avaliação da utilização de inaladores em pó de acordo com as características dos pacientes

\begin{tabular}{|c|c|c|c|}
\hline Características & Antes & Após & P-valor \\
\hline Toda a amostra & $4 \pm 1$ & $6 \pm 1$ & $<0,001$ \\
\hline \multicolumn{4}{|l|}{ Sexo } \\
\hline Feminino & $4 \pm 1,5$ & $6 \pm 1$ & $<0,001$ \\
\hline Masculino & $5 \pm 0,8$ & $6 \pm 0,8$ & 0,025 \\
\hline \multicolumn{4}{|l|}{ Idade } \\
\hline Menos de 60 anos & $4 \pm 1$ & $6 \pm 0,5$ & $<0,001$ \\
\hline 60 anos ou mais & $4 \pm 3$ & $6 \pm 1,8$ & $<0,001$ \\
\hline \multicolumn{4}{|l|}{ Escolaridade } \\
\hline Menos de 8 anos & $4 \pm 3$ & $6 \pm 2$ & $<0,001$ \\
\hline 8 anos ou mais & $5 \pm 1$ & $6 \pm 0$ & 0,006 \\
\hline \multicolumn{4}{|l|}{ Doença de base } \\
\hline Asma & $4,5 \pm 1$ & $6 \pm 1$ & $<0,001$ \\
\hline Controlada & $5 \pm 1$ & $6 \pm 0,5$ & 0,007 \\
\hline $\begin{array}{l}\text { Parcialmente } \\
\text { controlada }\end{array}$ & $4 \pm 2,5$ & $\begin{array}{c}5,5 \pm \\
18\end{array}$ & 0,005 \\
\hline Mal controlada & $5 \pm 0,5$ & $6 \pm 0$ & 0,018 \\
\hline DPOC & $4 \pm 3$ & $6 \pm 2$ & 0,022 \\
\hline GOLD A ou B & $3 \pm 3$ & $5 \pm 2$ & 0,037 \\
\hline GOLD C ou D & $5 \pm 2,5$ & $6 \pm 0,5$ & 1,000 \\
\hline \multicolumn{4}{|l|}{ Tempo de tratamento } \\
\hline Menos de 10 anos & $4 \pm 2$ & $6 \pm 1$ & $<0,001$ \\
\hline 10 anos ou mais & $4 \pm 1$ & $6 \pm 1$ & $<0,001$ \\
\hline \multicolumn{4}{|l|}{$\begin{array}{l}\text { Demonstração na } \\
\text { prescricão }\end{array}$} \\
\hline Não & $4 \pm 0$ & $6 \pm 0,5$ & 0,048 \\
\hline Sim & $4,5 \pm 2,5$ & $6 \pm 1$ & $<0,001$ \\
\hline
\end{tabular}

Nota: Os p-valores referem-se ao teste de Wilcoxon para amostras pareadas

Tabela 3 - Escores de avaliação da utilização de inaladores pressurizados de acordo com as características dos pacientes

\begin{tabular}{|c|c|c|c|}
\hline Características & Antes & Após & P-valor \\
\hline Toda a amostra & $0 \pm 8$ & $5 \pm 6$ & $<0,001$ \\
\hline \multicolumn{4}{|l|}{ Sexo } \\
\hline Feminino & $-1 \pm 8$ & $4 \pm 6$ & $<0,001$ \\
\hline Masculino & $3 \pm 6,5$ & $6 \pm 3,8$ & $<0,001$ \\
\hline \multicolumn{4}{|l|}{ Idade } \\
\hline Menos de 60 anos & $1,5 \pm 6,8$ & $6,5 \pm 6,8$ & $<0,001$ \\
\hline 60 anos ou mais & $-1 \pm 9$ & $4 \pm 5$ & $<0,001$ \\
\hline \multicolumn{4}{|l|}{ Escolaridade } \\
\hline Menos de 8 anos & $-1,5 \pm 7,3$ & $4 \pm 6$ & $<0,001$ \\
\hline 8 anos ou mais & $2 \pm 8$ & $6 \pm 7$ & $<0,001$ \\
\hline \multicolumn{4}{|l|}{ Doença de base } \\
\hline Asma & $-0,5 \pm 9,5$ & $5,5 \pm 6,8$ & $<0,001$ \\
\hline Controlada & $3 \pm 9$ & $7 \pm 6$ & $<0,001$ \\
\hline $\begin{array}{l}\text { Parcialmente } \\
\text { controlada }\end{array}$ & $-2 \pm 5$ & $2,5 \pm 8$ & $<0,001$ \\
\hline Mal controlada & $1 \pm 7$ & $4 \pm 7$ & $<0,001$ \\
\hline DPOC & $0 \pm 5,5$ & $5 \pm 4$ & $<0,001$ \\
\hline GOLD A ou B & $-1 \pm 6,5$ & $5 \pm 5,5$ & 0,006 \\
\hline GOLD C ou D & $2,5 \pm 5,3$ & $5 \pm 2,5$ & 0,022 \\
\hline \multicolumn{4}{|l|}{$\begin{array}{l}\text { Tempo de } \\
\text { tratamento }\end{array}$} \\
\hline Menos de 10 anos & $0 \pm 8,3$ & $5,5 \pm 6,3$ & $<0,001$ \\
\hline 10 anos ou mais & $-1 \pm 7$ & $4 \pm 6$ & $<0,001$ \\
\hline \multicolumn{4}{|l|}{$\begin{array}{l}\text { Demonstração na } \\
\text { prescrição }\end{array}$} \\
\hline Não & $-2,5 \pm 7,5$ & $4 \pm 5,8$ & 0,001 \\
\hline Sim & $0 \pm 6,5$ & $5,5 \pm 7$ & $<0,001$ \\
\hline
\end{tabular}

Nota: Os p-valores referem-se ao teste de Wilcoxon para amostras pareada 
A Figura 3 mostra o aumento significativo no percentual de pontuações máximas dos IPos $(p<0,001)$ e IPre $(p=0,005)$, após intervenção. Os usuários de IPo apresentaram, ainda, maior proporção de escores máximos antes e após a intervenção.

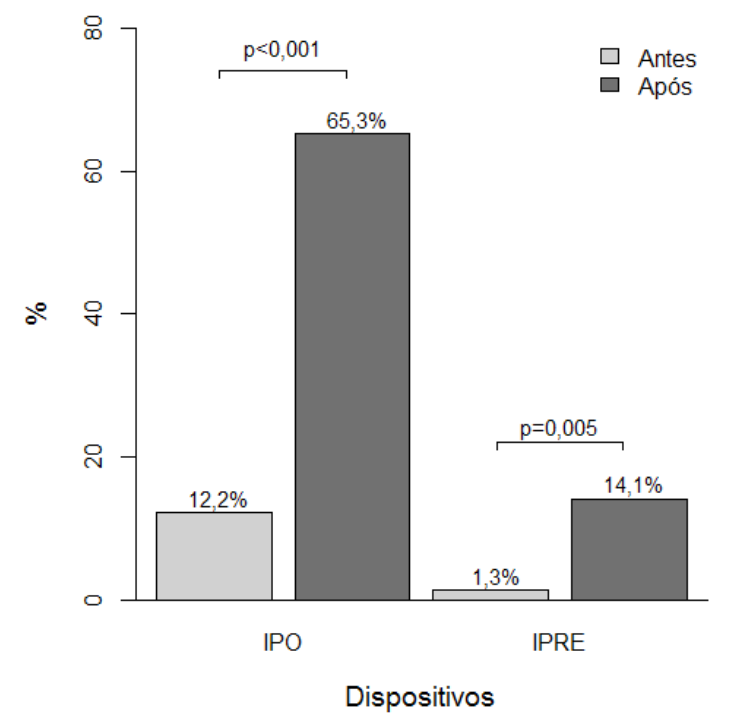

Nota: Os p-valores referem-se ao teste exato de Fisher. IPO = inaladores em pó; IPRE = inaladores pressurizados Pontuações mínima e máxima: inaladores em pó -6 e 6, inaladores pressurizados -18 e 9 .

Figura 3 - Indivíduos com pontuação máxima (não cometeram nenhum erro de utilização) por tipo de dispositivo, antes e após a intervenção

Quando comparados os dois dispositivos, foi observado que $70,8 \%$ dos pacientes que faziam uso de IPo aumentaram o escore de utilização, após a intervenção, e entre os usuários de IPre este percentual foi de $91 \%$ $(p=0,006)$. Não houve correlação significativa entre a melhora observada e as características dos pacientes.

Observa-se que a prevalência de algum erro entre os usuários de IPo é de $88,8 \%$ e, entre os usuários de IPre, de $98,7 \%$. Na população estudada, 120 testes apresentaram algum erro antes da intervenção, do total de 127 realizados, correspondendo a prevalência geral de erros de $94,5 \%$.

\section{DISCUSSÃO}

A pontuação máxima nos escores de avaliação da correção de técnica de uso de inaladores foi alcançada no momento inicial, antes da intervenção, por apenas 9,5\% $(n=11)$ da amostra, sendo 10 testes de IPo $(17,9 \%$ dos testes de IPo) e 1 de IPre (1,7\% dos testes de IPre). Esses números representam algum erro de técnica em 76,1\% dos pacientes usuários de IPo e de 98,2\% de IPre. Estes valores se assemelham aos de outros estudos ${ }^{4,5,7}$, que apontavam de
$51 \%$ a $82 \%$ de erros nos IPo e $71 \%$ a $94 \%$ de erros nos IPre. Em comparação com o estudo de Oliveira ${ }^{4}$, realizado no sul do Brasil, a prevalência geral de erros foi bastante aproximada. Enquanto nosso achado foi de $90,5 \%$, o estudo mostrou algum erro em $87 \%$ dos 110 testes realizados.

O estudo Global Initiative for Chronic Obstructive Lung Disease (GOLD) - Iniciativa Global para DPOC (tradução livre) - na última versão, divulgada em $2017^{9}$, aponta uma prevalência de erros em todos os DI's aproximadamente de $77 \%$, considerando pacientes portadores de DPOC que acabaram de sair do hospital. A elevação desse dado é corroborada por outro estudo, que reforça o achado de melhor técnica entre aqueles que saem do hospital com a prescrição em relação àqueles pacientes de regime ambulatorial ${ }^{9}$.

O Global Iniciative for Asthma ${ }^{10}$ - Iniciativa Global para Asma (tradução livre) - traz dados semelhantes aos do GOLD, de 70-80\% de ineficácia da técnica, enaltecendo ainda um dado importante, ou seja, os próprios prescritores desconhecem a técnica de uso dos inaladores. Assim sendo, não sabem também instruir os seus pacientes a usá-los corretamente, o que contribui diretamente para os dados apresentados.

Esse dado relacionado aos prescritores foi confirmado, indicando que a prevalência de erros semelhantes entre os médicos generalistas e a população usuária desses medicamentos é, atualmente, em torno de $87 \%$. Em contraste, médicos pneumologistas tiveram acerto em $62 \%$ dos casos, dado idêntico ao apresentado por farmacêuticos ${ }^{9}$. Ainda foi demonstrado que essa prevalência espelha a observada em internos de medicina, que só apresentaram a técnica correta em $5 \%$ dos casos ${ }^{3}$.

A diferença na distribuição de testes com pontuação máxima em relação aos dispositivos se apresentou significativa, no sentido de que pacientes usuários de IPo têm maior índice de técnica correta em relação aos usuários de IPre, o que foi observado tanto antes quanto depois da intervenção $(17,9 \%$ versus $1,7 \%$ antes da intervenção; $75 \%$ versus $18,3 \%$ depois da intervenção). Essa relação também se aproxima a do estudo de Oliveira, que mostrou acerto maior nos usuários de IPo sobre os de IPre (49\% versus $28 \%)^{4}$. Cabe ressaltar que os critérios utilizados para pontuar as técnicas foram diferentes, mas que a relação entre IPre e IPo se mantém.

Tal dado se contrapõe à prevalência de usuários de IPo $(58,3 \%)$ e de IPre $(76,4 \%)$, já que o tipo de dispositivo com maior índice de erros é também o mais utilizado, como apontado por outros estudos ${ }^{1,3,4,5,7,10}$. Parte dessa diferença pode ser atribuída ao fato de o tipo de dispositivo da droga de resgate mais difundida, o salbutamol, ser disponível somente em dispositivos pressurizados.

O confronto - intervenção proposta - entre a técnica cotidiana e a técnica correta para o paciente trouxe benefícios significativos na correção da técnica, independentemente da maioria dos fatores avaliados. Os 
Garib JR, et al. Avaliação da técnica de uso de dispositivos inalatórios no controle ambulatorial de asma.

pacientes com melhora não significativa apresentaram tendências que, provavelmente, se comprovariam com estudos de maior porte.

Os padrões de melhora foram diferentes para os dispositivos. Enquanto nos testes de IPo houve mais pontuações máximas após a intervenção; nos testes IPre a quantidade de pacientes com alguma melhora de técnica foi maior, mesmo que não alcançando a pontuação máxima.

Logo, apesar de em ambas ter sido observada alguma melhora, seria mais fácil alcançar pontuações máximas utilizando IPo do que IPre. Esse fato se justifica pelo maior número de etapas e pela necessidade de coordenação entre elas exigida no IPre, ilustrada por um maior número de momentos avaliados no escore utilizado.

Ao se avaliar esse padrão de aprendizagem, deve-se ter o cuidado de se observar que ele é de melhor qualidade no IPo, pois mais indivíduos alcançam pontuação máxima nesse processo ( $82 \%$ versus $17,2 \%$ ) e de maior quantidade no IPre, já que mais indivíduos apresentaram alguma melhora na sua pontuação $(96,7 \%$ versus $69,6 \%)$. Vale ressaltar ainda que o índice de pacientes sem melhora alguma na pontuação foi maior no IPo, apesar de não ter sido realizada análise estatística desse dado.

A pontuação inicial nos usuários de IPo mostrou-se independente dos fatores avaliados (sexo, idade, tempo de tratamento, doença de base, escolaridade e demonstração na primeira prescrição). Por outro lado, observou-se uma tendência para melhora da pontuação inicial em pacientes com mais de 8 anos de escolaridade, mas não houve comprovação estatística.

$\mathrm{Na}$ avaliação dos dispositivos IPre, o cenário foi semelhante e os fatores se mostraram, em sua maioria, independentes em relação à pontuação inicial. No entanto, a tendência a melhores pontuações foi observada no sexo masculino, mas sem significância. $\mathrm{O}$ único fator que se mostrou significativo de fato para melhores pontuações iniciais foi a demonstração no momento da prescrição.

Portanto, pode-se dizer que os resultados ressaltaram ainda a importância da demonstração no momento da primeira prescrição. O aumento da pontuação foi significativo em pacientes usuários de IPo que contaram com demonstração na primeira prescrição; enquanto aqueles que não tiveram esta oportunidade apresentaram tendência, ainda que não significativa, à melhora da pontuação.

Nos usuários de IPre, este fator se tornou ainda mais crucial. A demonstração no momento da primeira prescrição mostrou-se de fato significativa para maiores escores tanto antes quanto depois da intervenção, diferentemente dos pacientes usuários de IPo. Com isso, observa-se que os pacientes usuários de IPre que contam com essa oportunidade têm maiores chances tanto de apresentar melhores resultados em domicílio (representado pelo escore antes da intervenção) quanto de aprender de forma mais eficaz a técnica correta.
Avalia-se que este cuidado na primeira prescrição traria menor número de vícios de uso, de difícil correção após um período transcorrido de tratamento. Dessa forma, aconselha-se tornar essa demonstração imperativa na primeira prescrição de qualquer dispositivo inalatório, pois se observa que a pontuação inicial do paciente seria melhor, assim como a sua capacidade de aprendizagem. Além disso, o confronto entre a técnica domiciliar e a técnica correta de uso deve ser realizado periodicamente para reavaliação da técnica do paciente - como demonstrado pelas principais diretrizes de manejo de $\mathrm{DPOC}^{9}$ e $\mathrm{Asma}^{10}$ - já que mais de 90\% da amostra apresentou algum erro, e 83,6\% tiveram melhora objetiva da sua pontuação.

Apesar de creditar a falta desta demonstração ao curto tempo disponível para cada consulta, o tempo médio de cada teste propriamente foi estimado em cerca de 5 minutos. Pelos benefícios da técnica apresentados por este confronto e o que ele traduz para o desenvolvimento clínico do paciente e também para o aspecto financeiro do seu tratamento, é injustificável não se realizar este tipo de demonstração em períodos determinados. Não foi encontrado estudo que mensurasse o tempo desta intervenção para efetiva comparação.

Não há estudos atualmente que apontem um protocolo para revisões de técnica, indicando os momentos ideais para que sejam realizados. No entanto, aconselhase que ela seja realizada quando não há resposta clínica esperada com a introdução de uma nova droga ou quando há uma piora importante do quadro do paciente, antes de se prescrever uma nova droga.

A diretriz internacional para controle do DPOC ainda ressalta que uma avaliação do uso correto do dispositivo inalatório deveria ser feita em cada consulta, mas não especifica a forma que deve ser adotada para essa verificação ${ }^{11}$.

$\mathrm{O}$ encontro de diferença não estatisticamente significativa $(p>0,05)$ nos pacientes com DPOC subclassificados quanto ao risco de obstrução segundo GOLD (vide Anexo) pode se justificar pelo menor número de indivíduos em cada categoria, já que foi observada diferença estatisticamente significativa, quando se avalia os portadores de DPOC como um todo, com uma amostra maior de pacientes. Em todos os estudos analisados ${ }^{1,4,5,7}$, apontava-se para deficiente técnica independente da doença de base, mas não houve proposta de intervenção que mostrasse resultado semelhante de melhora não significativa de acordo com os subgrupos.

Apesar de erros não terem sido mensurados especificamente, vários deles eram cometidos, sendo os mais recorrentes a ausência de expiração profunda antes da inspiração da medicação; o disparo do IPre sem inspiração profunda logo em seguida; não manter a capacidade pulmonar total por tempo suficiente após disparo e profundidade de inspiração insuficiente.

Tal percepção vai ao encontro dos achados de 
Garib JR, et al. Avaliação da técnica de uso de dispositivos inalatórios no controle ambulatorial de asma.

Oliveira $^{4}$, que mensurou no sul do Brasil que em ambos os dispositivos o erro mais comum no processo era o de não expirar corretamente antes da administração do jato. $\mathrm{O}$ apontado seria de $66 \%$ para os IPo e de $47 \%$ para os IPre. Esse estudo apresentou como segundo erro mais comum a não manutenção da capacidade pulmonar total após a inalação (29\% para IPo, 25\% para IPre) e de não agitar o canister do IPre antes de utilizá-lo em 21\%.

No presente estudo, ainda foram observados erros de maior gravidade, como disparo do dispositivo em região muito distante da boca (ex.: braços, tronco), respiração nasal, ausência de preparo da dose tanto no IPre quanto no IPo, que desperdiçam o uso da medicação, em efeito semelhante ao apontado por Rau ${ }^{11}$.

Segundo esse estudo, a proporção de pacientes que não têm qualquer efeito da medicação pode chegar a $68 \%$, o que traria um rombo grande na distribuição destes, estimado nos Estados Unidos em até 15,5 bilhões de dólares ${ }^{9}$. Essa estimativa ainda não foi feita no Brasil.

O estudo apresentou limitações importantes que, apesar de não invalidarem os resultados encontrados, devem ser demarcadas, dentre elas a consideração de unidade amostral como teste, e não como indivíduo, tendo em vista que cada pessoa pode ter fatores imutáveis que

\section{REFERÊNCIAS}

1. Ganguly A, Das AK, Roy A, Adhikari A, Banerjee J, Sen S. Study of proper use of inhalational devices by bronchial asthma or COPD patients attending a tertiary care hospital. J Clin Diagn Res. 2014;8:HC04-7. doi: 10.7860/ JCDR/2014/9457.4976

2. Mascarenhas J, Bettencourt P, Azevedo A. Clinical epidemiology of chronic obstructive pulmonary disease. Arq Med. 2011;25(4):146-52. Disponível em: http://www.scielo. mec.pt/pdf/am/v25n4/v25n4a03.pdf.

3. Price D, Bosnic-Antitevich S, Briggs A, Chrystyn H, Rand $\mathrm{C}$, Sheuch $\mathrm{G}$, et al. Inhaler competence in asthma: Common errors, barriers to use and recommended. Respir Med. 2013;107:37-46. doi: 10.1016/j.rmed.2012.09.017.

4. Oliveira PD, Menezes AMB, Bertoldi AD, Wehrmeister FC, Macedo SEC. Assessment of inhaler techniques employed by patients with respiratory diseases in southern Brazil: a population-based study. J Bras Pneumol.2014;40(5):513-20. doi: http://dx.doi.org/10.1590/S1806-37132014000500007.

5. Khassawneh BY, Al-Ali MK, Alzoubi KH, Batarseh MZ, Al-Safi SA, Sharara AM, et al. Handling of inhaler devices in actual pulmonary practice: metered-dose inhaler versus dry powder inhalers. Respir Care. 2008;53(3):324-8. Available from: http://rc.rcjournal.com/content/respcare/53/3/324. full.pdf.

6. Zambelli-Simões L, Martins MC, Possari JCC, Carvalho GB, comprometem o teste, tendo um "peso duplo" na análise estatística. Durante a recuperação dos dados, ficou clara a necessidade de ter sido realizada separação da frequência para cada dispositivo, nos casos em que o paciente fez uso de dois ou mais da mesma classe (IPo ou IPre), configurando-se como um viés de registro das informações.

O uso de nebulizadores e espaçadores, não foi considerado nesse estudo, mas em tese, têm distribuição mais fácil, e, apesar disso, ainda se tem demonstrado que há grande proporção de erros com o uso deles, chegando a até $57 \%$ dos pacientes 9 . Ainda há de ressaltar que nem todos que cometem algum erro não têm benefício da droga, já que em algumas situações se perde alguma efetividade, mas não toda ela.

A técnica preconizada para alcançar a biodisponibilidade aceitável dos dispositivos inalatórios não é simples, portanto a orientação durante a primeira prescrição deve sempre ser enfatizada e reavaliada periodicamente com o paciente durante o acompanhamento. Essa abordagem traz impactos clínicos e econômicos positivos, afinal um paciente em uso correto da medicação e com a doença controlada, apresenta menor incidência de exacerbações e não necessita de aumentos desnecessários da medicação.

Coelho ACC, Cipriano SL, et al . Validation of scores of use of inhalation devices: valoration of errors. J Bras Pneumol. 2015;41(4):313-22. doi: http://dx.doi.org/10.1590/S180637132015000004435 .

7. Arora P, Kumar L, Vohra V, Sarin R, Jaiswal A, Puri MM et al. Evaluating the technique of using inhalation device in COPD and bronchial asthma patients. Respir Med. 2014;108(7):9928. doi: 10.1016/j.rmed.2014.04.021.

8. Sanchis J, Corrigan C, Levy ML, Viejo JL. Inhaler devices from theory to practice. Respir Med 2013;107:495-502. doi: 10.1016/j.rmed.2012.12.007.

9. Global Strategy for the Diagnosis. Management and Prevention of COPD. Global Initiative for Chronic Obstructive Lung Disease (GOLD) 2017. Available from: http://goldcopd. org.

10. Global Initiative for Asthma. Global Strategy for Asthma Management and Prevention, 2017. Available from: www. ginasthma.org.

11. Rau JL. Practical problems with aerosol therapy in COPD. Respir Care. 2006;51(2):158-72. Available from: http:// rc.rcjournal.com/content/respcare/51/2/158.full.pdf.

12. IV Diretrizes Brasileiras para o Manejo da Asma. J Bras Pneumol. 2006;32(Supl 7):S 447-S474. doi: http://dx.doi. org/10.1590/S1806-37132006001100002. 
Garib JR, et al. Avaliação da técnica de uso de dispositivos inalatórios no controle ambulatorial de asma.

Participação dos autores: Junia Rios Garib: seleção de pacientes, redação do artigo, elaboração do projeto, orientação do estudo, análise dos resultados. Beatriz Carvalho Martins Brandão Leite: seleção de pacientes, redação do artigo, elaboração do projeto, realização de exame clínico, preenchimento dos questionários, coleta de dados. Victor Chaves Reis: seleção de pacientes, redação do artigo, elaboração do projeto, realização de exame clínico, preenchimento dos questionários, coleta de dados. Marcelo Leonardo Araujo Campos: seleção de pacientes, redação do artigo, realização de exame clínico, preenchimento dos questionários, coleta de dados. Sarah Veiga Medrado: seleção de pacientes, redação do artigo, realização de exame clínico, preenchimento dos questionários, coleta de dados. Flávio Mendonça Andrade da Silva: seleção de pacientes, redação do artigo, elaboração do projeto, orientação do estudo, análise dos resultados.

Recebido em: 24.10 .17

Aceito em: 02.02.18 